\title{
PARP inhibitors: review of mechanisms of action and BRCA1/2 mutation targeting
}

\author{
Karolina N. Dziadkowiec ${ }^{1}$, Emilia Gąsiorowska ${ }^{1}$, Ewa Nowak-Markwitz ${ }^{1}$, Anna Jankowska ${ }^{2}$ \\ ${ }^{1}$ Department of Gynaecological Oncology, Poznan University of Medical Sciences, Poznan, Poland \\ ${ }^{2}$ Department of Cell Biology, Poznan University of Medical Sciences, Poznan, Poland
}

\begin{abstract}
Poly(ADP-ribose) polymerases have shown true promise in early clinical studies due to reported activity in BRCA-associated cancers. PARP inhibitors may represent a potentially important new class of chemotherapeutic agents directed at targeting cancers with defective DNA-damage repair. In order to widen the prospective patient population that would benefit from PARP inhibitors, predictive biomarkers based on a clear understanding of the mechanism of action are required. In addition, a more sophisticated understanding of the toxicity profile is required if PARP inhibitors are to be employed in the curative, rather than the palliative, setting. PARP inhibitors have successfully moved into clinical practice in the past few years, with approval granted from the Food and Drug Administration (FDA) and European Medicines Agency (EMA) within the past two years. The United States FDA approval of olaparib applies to fourth-line treatment in germline BRCA-mutant ovarian cancer, and European EMA approval of olaparib for maintenance therapy in both germline and somatic BRCA-mutant platinum-sensitive ovarian cancer. This review covers the current understanding of PARP, its inhibition, and the basis of the excitement surrounding these new agents. It also evaluates future approaches and directions required to achieve full understanding of the intricate interplay of these agents at the cellular level.
\end{abstract}

Key words: PARP, BRCA, mutation, olaparib, ovarian cancer, poly(ADP-ribose) polymerases, DNA, PARP1, PARP2.

\section{Introduction}

Breast cancer is the most frequently occurring cancer in women worldwide; ovarian cancer is the second most common gynaecological cancer in developed countries such as Poland, the United States, and the United Kingdom [1]. Approximately 14,180 women die from ovarian cancer and 40,290 women die from breast cancer yearly [1]. The overall five-year survival rate is $36 \%$ and $80 \%$ for ovarian and breast cancer, respectively [1].

The especially high degree of fatality of ovarian cancer may be correlated to its ambiguous, nonspecific, and often asymptomatic course. Alarming symptoms usually appear when the disease has advanced outside the ovary. The identification of early-stage ovarian cancer confined to the ovary is often incidental and carries a five-year survival of $92 \%$ [2]. The mortality rates from ovarian cancer have remained largely unchanged for over five decades [3].

Breast cancer incidence has shown the highest rates in developed countries, urban populations, and in Caucasian women. The highest incidence occurs in
North America and the lowest in Asia [4]. It has been shown that BRCA1 gene mutations account for $1-2 \%$ of breast cancers and virtually all familial breast-ovary tumours [5]. The prognosis of breast cancer is determined through several characteristic features, namely, oestrogen (OR), progesterone (PR), and HER2 receptor status and mutation status. BRCA1 mutations usually confer a more aggressive phenotype, are high grade, and are more likely to be triple-negative (OR, PR, and HER2). BRCA2 mutations resemble sporadic breast cancer [6].

This review will summarise the recent development of poly(ADP-ribose) polymerases (PARP) as new emerging agents in the treatment of tumours with BRCA and BRCA-related mutations.

\section{DNA damage repair pathways and BRCA function}

The past few years have brought dramatic advances in our understanding of the mechanism and regulation of cellular components that are of crucial importance in the repair processes of DNA damage. DNA encounters various assaults on its native structure and sequence 
throughout the life span of a cell [8]. Human cells have at least five primary pathways of DNA repair, which are systems that serve to probe and identify defects protecting the genome. The major DNA repair pathways are direct repair, mismatch repair (MMR), base excision repair (BER), nucleotide excision repair (NER), and double-strand break (DSB) recombinational repair, which includes both non-homologous end-joining (NHEJ) and homologous recombinational repair [7]. Dysfunction, reduction, or absence of proteins committed to these pathways may lead to disastrous cellular consequences causing mutagenesis and toxicity.

In recent years, BRCA1 and BRCA2 tumour suppressor genes have been linked to a fundamental role in the response to cellular damage through activation of specific DNA repair processes. Both the BRCA1 and BRCA2 proteins are often found in stable interaction, suggesting these proteins cofunction in pathways of tumour suppression. Both genes have been proposed to function in DNA repair and as transcriptional regulators. BRCA1 and BRCA2 form a complex with Rad51, a protein that has an established role in homologous recombination [9].

It has been shown that BRCA1 is also involved in complexing with and activation of p53 [11]. The tumour suppressor protein $\mathrm{p} 53$ is involved in a variety of human cancers [10]; the normal function of p53 is to signal the occurrence of DNA damage and temporarily arrest the cell cycle to either allow repair or trigger cell death. A more detailed analysis of the effects of BRCA genes and their transcriptional functions may result in a clearer understanding of their tissue-specific actions.

\section{BRCA mutations and cancer risk}

There is a clearly established association of germline mutations in BRCA1 and BRCA2 and the development of breast or ovarian cancer syndrome [12]. BRCA1 and BRCA2 gene mutations are notably linked to inherited breast and ovarian cancers, and are also implicated in sporadic malignancies. These genes can therefore be associated with the development of tumours with mutations derived from either germline or somatic (tumour only) variants [13].

The current methods used for the identification of BRCA gene mutations is dependent on DNA sequencing techniques. Currently, one of the difficulties with this method is differentiating between clinically significant changes and benign non-pathogenic variations in these genes, termed variants of unknown significance (VUS). Genetic testing has revealed that approximately $13 \%$ of BRCA1 and BRCA2 mutations are VUS, implying clinical uncertainty and ambiguity in risk assessment of tested individuals [14, 15]. Evidently, the task of accurately identifying carriers of BRCA mutations is complicated by our continued lack of understanding of the signifi- cance of various polymorphisms in these genes and the mechanism of tumorigenesis conferred upon mutation.

It has been recognised that BRCA1-related breast cancers are more likely to be ER-negative than are BRCA2 and non-BRCA1 cancers [16]. Oestrogen has a profound effect on both normal and malignant cells. It is known that certain genes controlling growth regulation are heavily influenced by the effects of oestrogen. Breast and ovarian cancers are often initially evaluated for oestrogen receptor (ER) status, with the rationale of individualised therapy. Knowledge of ER status provides additional information with regard to patient prognosis and treatment directives. The failures of BRCA function and oestrogen signalling among other mechanisms promotes a lack of proper DNA surveillance, leading to tumorigenesis. It has been shown that gene silencing of BRCA1 is associated with increased gene expression of aromatase catalysing the conversion of steroids into active oestrogens. This results in increased activity and therefore increased oestrogen synthesis [17]. Thorough evaluation of oestrogen signalling is required to provide the effective preventive, therapeutic, and possibly curative measures in both BRCA and non-BRCA cancers.

\section{Poly(ADP-ribose) polymerase (PARP) activity and inhibition}

Poly(ADP-ribose) polymerases (PARPs) constitute a large family of 18 proteins [18]. PARP1 and PARP2 are enzymes, activated by DNA damage, which facilitate DNA repair in pathways involving single-strand breaks (SSBs) and base excision repair (BER). PARP1 behaves as a 'DNA nick' signalling SSB where it is recruited and participates in DNA repair [19]. PARP1 becomes activated upon detection and binding to areas of single strand damage via its zinc-finger DNA-binding domain. After binding to altered DNA, PARP1 increases its catalytic activity and uses $\mathrm{NAD}^{+}$to create polymers of poly(ADPribose) (PAR) and transfers it to acceptor proteins, including PARP itself [20]. This auto-poly(ADP-ribosyl) ation recruits various other proteins to the site of DNA-damage, initiating a repair complex. Eventually, PARP1 undergoes a molecular change that ultimately leads to its reduced affinity for DNA; following its release the damaged site may be accessed by other repair complex proteins [20]. Additionally, overactivation of PARP1 induces reduction of $\mathrm{NAD}^{+}$and ATP, resulting in cellular dysfunction and may lead to necrosis or apoptosis [21].

\section{Clinical application of PARP inhibitors in BRCA mutations}

DNA replication and error-repair is a critical component of cancer cell survival. Briefly, suppression of PARP 
A

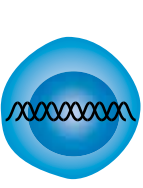

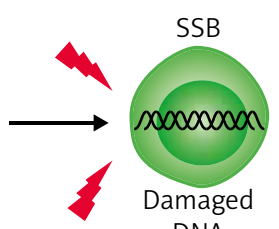

DNA

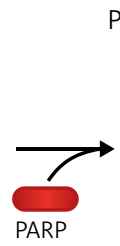

PARP recruitment

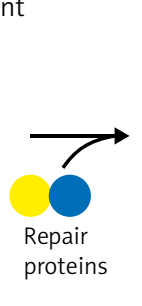

DNA repair

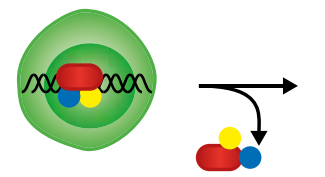

PARP and repair proteins dissociate from DNA

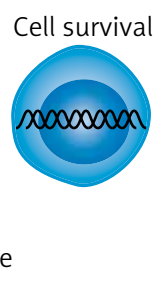

B

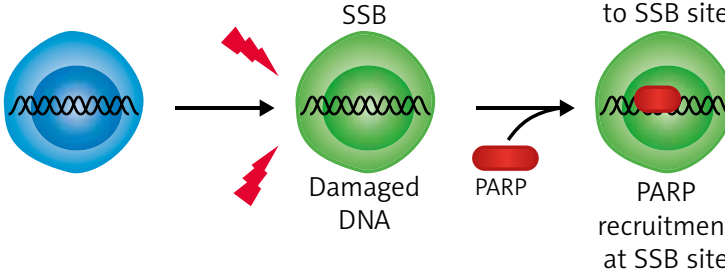

PARP recruitment

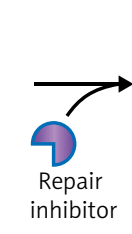

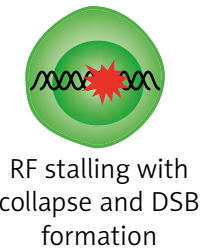

formation

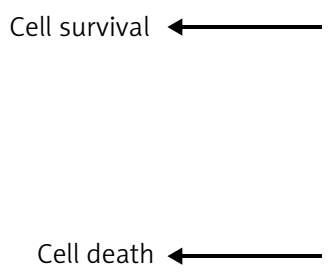

Cell survival

Cell death
DSB repair via $H R$ and RF restart

Impaired HR with DSB accumulation

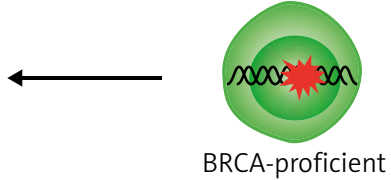

cell
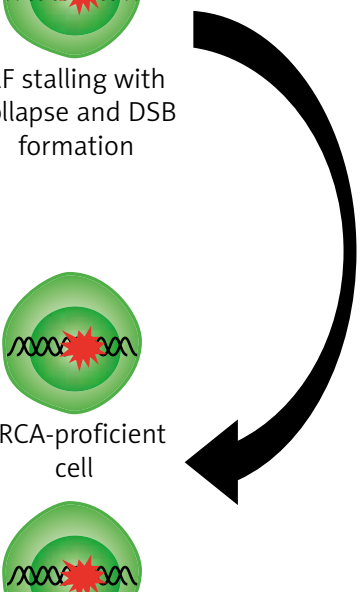

BRCA-deficient cell

$S S B$ - single-strand break; DSB - double-strand break; RF-replication fork; HR - homologous recombination

Fig. 1. A) Proper DNA repair mechanism with functional PARP protein and DNA repair proteins. B) Attempted DNA repair of SSB in the presence of PARP inhibitor resulting in DSB formation. BRCA-proficient cells have the ability to repair the DSB and restart; maintaining survival. BRCA-deficient cells are unable to repair the accumulating DSB's which inevitably results in cell death

leads to stalling of replication forks due to the accumulation of unrepaired SSBs [22]. Stalled replication forks degrade into highly cytotoxic double strand breaks (DSBs) if not corrected by appropriate repair mechanisms, which are essentially absent in BRCA-mutated cells [22]. Since BRCA-mutated cells are incapable of HR (considered the most precise DSB repair mechanism), PARP inhibition results in genomic instability and cell death [23]. Thus, tumours that harbour a defect in homologous repair (HR) (and likewise a defect in DSB repair) seem to be highly vulnerable to the effects of PARP inhibition. Notably, these tumours include somatic and germline BRCA-1 and BRCA-2 mutations.

The most recent reported clinical trials are testing the efficacy of currently available PARP inhibitors. The phase III trials of talazoparib, niraparib, olaparib, and veliparib have shown antitumor efficacy and tolerability and will continue to be researched. Olaparib has recently been granted accelerated approval based on clinical data showing drug effectiveness. The approval was granted under the notion that the drug is used to treat a life-threatening disease, and clinical trials will probably predict clinical benefit to patients. Limited data are available on the activity of olaparib, warranting further investigation.

Currently, all PARP inhibitors are believed to inhibit both PARP1 and PARP2 [23]. The suppression of PARP catalytic activity prevents the formation of PAR polymers and blocks the binding of $\mathrm{NAD}^{+}$at the site of DNA damage, ultimately compromising a cell's ability to overcome DNA-dependent damage [24]. Additional studies and trials are required to further our understanding of PARPs and their complete biological roles.

\section{Predicting response to PARP therapy}

The identification of a reliable biomarker of response to PARP inhibition has found to be exceptionally challenging due to inconsistency from various investigative approaches. Recent studies have revealed promising biomarkers through the measurement of PAR poly(ADP-ribose) levels, formation of RAD51 foci, and calculating the levels of 53BP1 expression in can- 
cer cells [25]. Hence, the identification of an accurate biomarker is imperative in order to optimise clinical responsiveness to PARP inhibitor therapy.

\section{PARP inhibitors and synthetic lethality}

PARP inhibitors have shown promise in oncology through synthetic lethality, among other applications. Synthetic lethality arises when two agents applied independently permit cell survival whereas both events in combination result in cell death. The greatest advantage of exploiting synthetic lethality is that tumour tissue is selectively targeted resulting in decreased toxicity to normal cells.

The inhibition of PARP prevents timely repair of persistent SSBs that culminate in degradation into double strand breaks (DSBs) when encountered by replication forks, which collapse and cannot be restarted [26]. Normal cells with a BRCA mutation are often heterozygous for the abnormal gene, are able to repair the DBSs, and survive unaffected under PARP inhibition. Conversely, cancer cells may be homozygous for the mutated $B R C A 1$ or $B R C A 2$, conferring inadequate DNA repair and susceptibility to the synthetic lethality of PARP inhibitors [27]. This clinical strategy potentially provides a wider variety of therapeutic possibilities, and this alone may even prove to be sufficient enough to avoid the toxic effects of chemotherapy and radiation.

\section{Safety of PARP inhibitors}

The toxicities of PARP inhibitors are comparable to other chemotherapeutic agents. Studies have revealed that nausea, fatigue, vomiting, anaemia, and abdominal pain are among the most frequently reported adverse effects [28]. A serious concern, as with all chemotherapeutic agents, is the development of de novo primary malignancies [29]. Continued research and follow-up are imperative to outline the risk and to achieve the full potential of PARP inhibitor therapy.

\section{Resistance to PARP inhibitors}

Resistance to PARP inhibitors poses a significant barrier to the long-term survival and treatment options of patients with BRCA1- and BRCA2-mutated cancers. A large fraction of patients with tumours that are potentially amenable to therapy either do not respond to PARP inhibitor treatment or rapidly develop clinical resistance. Acquired resistance is becoming a significant problem. It is largely unknown how resistance to PARP inhibitors develops. At present, there are a number of mechanisms believed to confer resistance to PARP inhibitors. Firstly, it is thought that a reversion mutation in the BRCA gene could potentially result in resistance by restoring homologous recombination (HR) function [30]. These newly resistant cancer cells with restored $H R$ function have a significant survival advantage and are therefore more resistant to treatment. Secondly, a decrease in non-homologous end-joining (NHEJ) may provide resistance to
PARP inhibitors. The NHEJ repair pathway is normally upregulated during PARP inhibition, playing an important role in synthetic lethality. Thirdly, decreased levels, activity, or enzymatic action of PARP-1 available for inhibition is pivotal for the effectiveness of PARP inhibitor treatment. Furthermore, upregulation of permeability glycoprotein efflux pumps results in increased PARP inhibitor efflux and decreased intracellular concentrations of the agents [31, 32]. Lastly, the increased activity of RAD51, an essential protein involved in $\mathrm{HR}$, results in resistance to PARP inhibitors [33].

Treatment of certain patients with PARP inhibitors may require knowledge of particular resistance mechanisms that could influence the efficacy of treatment. Therefore, the assessment of resistance mechanisms and controlling these factors prior to treatment with PARP inhibitors may provide better outcomes [34].

This class of agents has great potential for development; it is essential to understand more fully the mechanism and interplay of PARP inhibition and resistance before clearer conclusions can be defined.

\section{Conclusions and future directions}

Research into PARP inhibition has shown efficacy and the potential to directly target DNA repair mechanisms as an effective method of new anti-neoplastic agents. Several PARP inhibitors, including the ones mentioned above, are currently under extensive investigation and development. These agents have been shown to have similar toxicity profiles and adverse effects as other regularly used chemotherapeutics. Studies have shown the effectiveness of PARP inhibition as single agents and in combination with other chemotherapy strategies.

PARP inhibitors offer a promising strategy, but many questions apart from clinical efficacy remain unanswered. For instance, for the long-term effects of administration of PARP inhibitors or similar, it is not clear if these drugs potentiate other toxicities when used in combination.

At the same time, there is a continual demand and search for biomarkers that can efficiently identify tumours that are most likely to respond to PARP inhibitor treatment. These new agents appear likely to become a fundamental components in the management of patients with BRCA mutations associated tumours. Presently, research concerning other genetic defects that could potentially confer susceptibility to PARP inhibitor treatment is on-going. Expanding and identifying populations that could be treated with these new class of agents may result in improved clinical outcomes.

Overall, PARP inhibitors are an exciting new class of drugs that have attracted a great deal of attention and shown great potential for future development. Consistent research and progress of current studies will lead 
to a stronger understanding and a more comprehensive view of these agents and their role in future treatment strategies.

\section{Disclosure}

\section{Authors report no conflict of interest.}

\section{References}

1. Siegel RL, Miller KD, Jemal A. Cancer statistics, 2015. CA Cancer J Clin 2015; 65: 5-29.

2. Buys SS, Partridge E, Black A, et al. for the PLCO Project Team. Effect of screening on ovarian cancer mortality: the Prostate, Lung, Colorectal and Ovarian (PLCO) Cancer Screening Randomized Controlled Trial. JAMA 2011; 305: 2295-2303.

3. Howlader N, Noone AM, Krapcho M, et al. (eds.). SEER cancer statistics review, 1975-2011, National Cancer Institute [Internet]. National Cancer Institute, Bethesda 2014

4. Porter PL. Global trends in breast cancer incidence and mortality. Salud Pública de México 2009; 51 (suppl 2):s141-146.

5. Karami F, Mehdipour P. A comprehensive focus on global spectrum of BRCA1 and BRCA2 mutations in breast cancer. Biomed Res Int 2013; 2013: 928562.

6. Atchley DP, Albarracin CT, Lopez A, et al. Clinical and pathologic characteristics of patients with BRCA-positive and BRCA-negative breast cancer. J Clin Oncol 2008; 26: 4282-4288.

7. Plummer R. Perspective on the pipeline of drugs being developed with modulation of DNA damage as a target. Clin Cancer Res 2010; 16: 4527 4531.

8. Hansen WK, Kelley MR. Review of mammalian DNA repair and translational implications. J Pharmacol Exp Ther 2000; 295: 1-9.

9. Scully R. Role of BRCA gene dysfunction in breast and ovarian cancer predisposition. Breast Cancer Res 2000; 2: 324-330.

10. Hollstein M, Sidransky D, Vogelstein B, Harris C. p53 mutations in human cancers. Science 1991; 253: 49-53.

11. Ouchi T, Monteiro ANA, August A, et al. BRCA1 regulates p53-dependent gene expression. Proc Natl Acad Sci U S A 1998; 95: 2302-2306.

12. Alsop K, Fereday S, Meldrum C, et al. BRCA mutation frequency and patterns of treatment response in BRCA mutation-positive women with ovarian cancer: a report from the Australian Ovarian Cancer Study Group. J Clin Oncol 2012; 30: 2654-2663.

13. Hennessy BT, Timms KM, Carey MS, et al. Somatic mutations in brca1 and brca2 could expand the number of patients that benefit from poly (adp ribose) polymerase inhibitors in ovarian cancer. J Clin Oncol 2010; 28: 3570-3576.

14. Richter S, Haroun I, Graham TC, et al. Variants of unknown significance in BRCA testing: Impact on risk perception, worry, prevention and counseling. Ann Oncol 2013; 24 (Suppl 8): viii69-viii74.

15. Frank TS, Deffenbaugh AM, Reid JE, et al. Clinical characteristics of individuals with germline mutations in BRCA1 and BRCA2: Analysis of 10,000 individuals. J Clin Oncol 2002; 20: 1480-90.

16. Foulkes WD, Metcalfe K, Sun P, et al. Estrogen receptor status in BRCA1and BRCA2-related breast cancer: the influence of age, grade, and histological type. Clin Cancer Res 2004; 10: 2029-34.

17. Ghosh S, Lu Y, Katz A, Hu Y, Li R. Tumor suppressor BRCA1 inhibits a breast cancer-associated promoter of the aromatase gene (CYP19) in human adipose stromal cells. Am J Physiol Endocrinol Metab 2007; 292: 246-52.

18. Ame JC, Spenlehauer C, de Murcia G. The PARP superfamily. Bioessays 2004; 26: 882-893.

19. Heale JT, Ball AR, Schmiesing JA, et al. Condensin I interacts with the PARP-1-XRCC1 complex and functions in DNA single-strand break repair. Mol Cell 2006; 21: 837-848.

20. Plummer R. Poly(ADP-ribose) polymerase inhibition: a new direction for BRCA and triple-negative breast cancer? Breast Cancer Res 2011; 13 : 218.
21. Sodhi RK, Singh N, Jaggi AS. Poly(ADP-ribose) polymerase-1 (PARP-1) and its therapeutic implications. Vascul Pharmacol 2010; 53: 77-87.

22. Helleday T. The underlying mechanism for the PARP and BRCA synthetic lethality: clearing up the misunderstandings. Mol Oncol 2011; 5: 387393.

23. Rouleau M, Patel A, Hendzel MJ, et al. PARP inhibition: PARP1 and beyond. Nat Rev Cancer 2010; 10: 293-301.

24. Livarqhi L, Garber JE. PARP inhibitors in the management of breast cancer: current data and future prospects. BMC Med 2015; 13: 188.

25. Oplustilova L, Wolanin K, Mistrik M, et al. Evaluation of candidate biomarkers to predict cancer cell sensitivity or resistance to PARP-1 inhibitor treatment. Cell Cycle 2012; 11: 3837-3850.

26. Arnaudeau C, Lundin C, Helleday T. DNA double-strand breaks associated with replication forks are predominantly repaired by homologous recombination involving an exchange mechanism in mammalian cells. J Mol Biol 2001; 307: 1235-1245.

27. Tutt AN, Lord CJ, McCabe $\mathrm{N}$, et al. Exploiting the DNA repair defect in BRCA mutant cells in the design of new therapeutic strategies for cancer. Cold Spring Harb Symp Quant Biol 2005; 70: 139-148.

28. Domchek SM, Aghajanian C, Shapira-frommer R, et al. Efficacy and safety of olaparib monotherapy in germline BRCA1/2 mutation carriers with advanced ovarian cancer and three or more lines of prior therapy. Gynecol Oncol 2016; 140: 199-203.

29. Livraghi L, Garber JE. PARP inhibitors in the management of breast cancer: current data and future prospects. BMC Med 2015; 13: 188.

30. Sakai W, Swisher EM, Karlan BY, et al. Secondary mutations as a mechanism of cisplatin resistance in BRCA2-mutated cancers. Nature 2008; 451: 1116-1120.

31. Montoni A, Robu M, Pouliot E, Shah GM. Resistance to PARP-Inhibitors in Cancer Therapy. Front Pharmacol 2013; 4: 18.

32. Ganguly B, Dolfi SC, Rodriguez-Rodriguez L, et al. Role of Biomarkers in the Development of PARP Inhibitors. Biomark Cancer 2016; 8 (Suppl 1): 15-25.

33. Oplustilova L, Wolanin K, Mistrik M, et al. Evaluation of candidate biomarkers to predict cancer cell sensitivity or resistance to PARP-1 inhibitor treatment. Cell Cycle 2012; 11: 3837-3850.

34. Lord CJ, Ashworth A. The DNA damage response and cancer therapy. Nature 2012; 481: 287-294. 